\title{
PROCÉDÉ DE RÉDUCTION DE LA CORROSION PAR LE CHLORE ET DE L'ENCRASSEMENT DES FAISCEAUX DE TUBES D'USINES D'INCINÉRATION
}

\author{
K.-M. Allal*, F. Birkner** \\ Servithen - ICA Chemie
}

Le procédé ICA est une réponse au problème de la corrosion par le chlore et de l'encrassement des faisceaux tubulaires de chaudières et échangeurs de chaleur dans les usines d'incinération. Celui-ci est mis en œuvre par l'injection au niveau de l'air secondaire de combustion, d'une substance minérale appelée ICA 5000 . Outre son effet inhibiteur de corrosion, ce procédé permet de protéger le revêtement réfractaire de la chambre de combustion, de former un dépôt meuble sur les surfaces chauffantes, d'augmenter la capacité de traitement de l'unité, de diminuer le temps de nettoyage et par là même de réduire les coûts d'entretien.

The solution to the chlorine corrosion and the fouling problems of boiler and heat exchanger tubes in waste incineration plants is given by the use of ICA products. This is mainly based on the injection at the secondary combustion air entrance of a mineral substance called ICA 5000. Beside its corrosion inhibition effect, it yields the protection of the combustion chamber refractory coating, the formation on the heated surfaces of an easily removable ash, an incresae of the plant treating capacity, a decrease of the cleaning time and hence, a lowering of the operating costs.

\section{INTRODUCTION}

Les variations de la composition des déchets ainsi que les nouvelles législations en vigueur, ont imposé des températures plus élevées au niveau de la chambre de combustion des unités d'incinération d'ordures ménagères, avec pour conséquence une détérioration par le chlore des surfaces exposées aux hautes températures.

En effet, lors de la combustion, une quantité importante de chlorures métalliques se forme dans les gaz. Ces chlorures d'alcalis, alcalino-terreux et autres métaux (plomb, zinc) se retrouvent dans les fumées sous forme solide, liquide ou gazeuse. Au contact avec le dioxyde de soufre présent dans les gaz, il se forme des dépôts durs de sulfates métalliques ainsi que du chlore gazeux fortement corrosif.

Le procédé développé et breveté par la société ICA Chemie permet d'une part de réduire les effets de la corrosion en empêchant la formation de chlore libre et d'autre part de for- mer des dépôts meubles de cendre sur les surfaces de chauffe, entraînant une diminution significative du temps nécessaire au nettoyage des tubes et par là même des coûts d'entretien. Ce procédé est principalement basé sur l'injection d'une substance pulvérulente hautement active au niveau de l'air secondaire de combustion.

\section{MÉCANISME DU PHÉNOMÈNE DE CORROSION}

Afin de pouvoir agir efficacement, il est essentiel de bien comprendre le processus de corrosion.

En fait, deux phénomènes simultanés se produisent dans la chambre de combustion. D'une part, les chlorures métalliques à l'état vapeur vont se condenser partiellement au contact des réfractaires et des tubes de chaudière (ou d'échangeur thermique). D'autre part, les chlorures métalliques véhiculés par les fumées à l'état solide ou liquide vont se déposer dans un état proche de leur état de fusion sur les blocs surchauffeurs. Ces deux phénomènes sont le point de départ du processus de corrosion par le chlore et de formation d'un dépôt dur sur les surfaces de chauffe.

Ces phénomènes entraînent généralement la dégradation du revêtement réfractaire, la corrosion des surfaces de vaporisation dans le cas d'une chaudière ainsi que la corrosion au niveau du surchauffeur accompagné d'un encrassement massif de la surface externe des tubes.

\section{Dégradation du réfractaire}

Il a été observé qu'une partie des chlorures métalliques condensés sur les parois des réfractaires était absorbée au niveau des jointures des plaques réfractaires ${ }^{[1]}$, provoquant ainsi des fissurations et par là même des chemins favorisant l'accès du chlore vers les tubulures (figure I). En effet, des analyses qualitatives ont montré la présence de $\mathrm{PbCl}_{2}$ et $\mathrm{ZnCl}_{2}$ en particulier au niveau des colles utilisées pour la fixation des plaques.

Corrosion des surfaces de vaporisation de chaudière

Ce processus est déclenché par la présence de chlore gazeux 

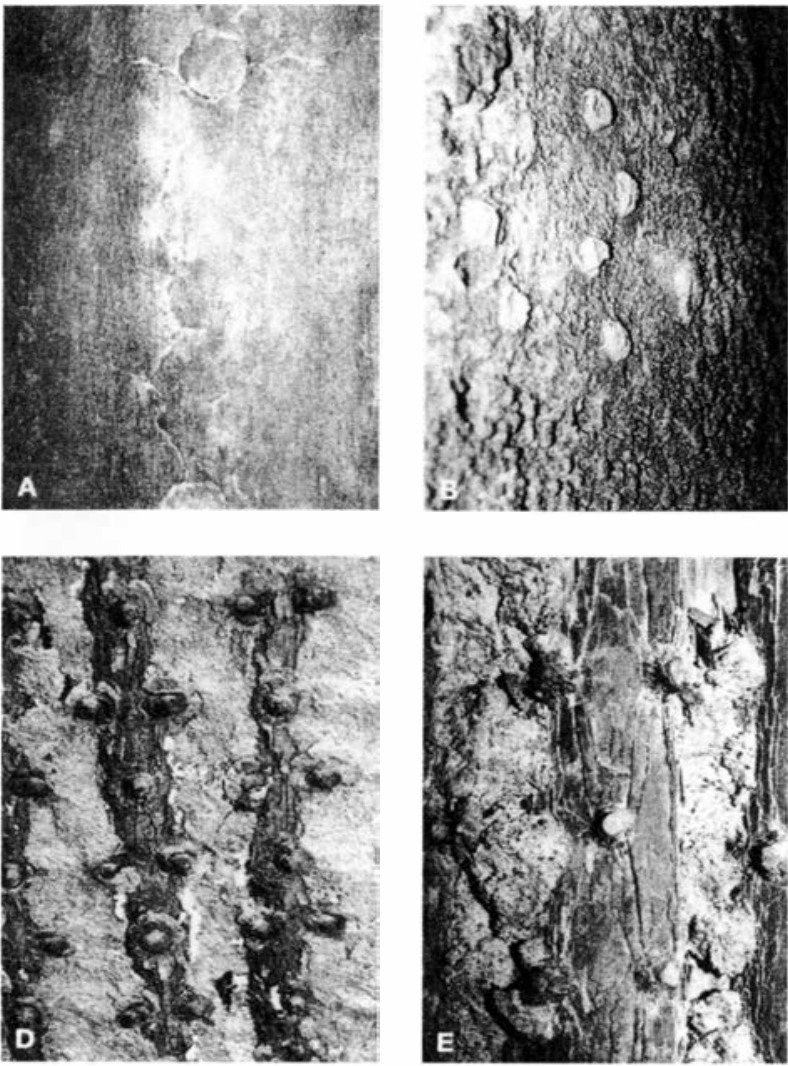

Figure I : Effet de la corrosion par le chlore sur les réfractaires et les tubes

dans les fumées. Celui-ci est généré par la réaction de sulfatation des chlorures métalliques selon la réaction I ciaprès :

$m \mathrm{MeCl}_{n}+(\mathrm{m} . \mathrm{n} / 2)\left[\mathrm{SO}_{2}(\mathrm{~g})+\mathrm{O}_{2}(\mathrm{~g})\right]$

$\longrightarrow \mathrm{Me}_{\mathrm{m}}\left(\mathrm{SO}_{4}\right)_{\mathrm{m} . \mathrm{n} / 2}+(\mathrm{m} . \mathrm{n} / 2) \mathrm{Cl}_{2}(\mathrm{~g})$

$\operatorname{avec} \mathrm{Me}=\mathrm{Na}, \mathrm{K}, \mathrm{Ca}, \mathrm{Fe}$, etc.

$\mathrm{n}=1,2,3,4$ (valence du métal)

$\mathrm{m}=1$ ou 2 suivant que $\mathrm{n}$ soit pair ou impair.

C'est en fait l'instabilité thermodynamique des chlorures à hautes températures ainsi que la présence de dioxyde de soufre dans les gaz de combustion qui engendrent la formation de chlore gazeux comme le montre clairement la figure 2.

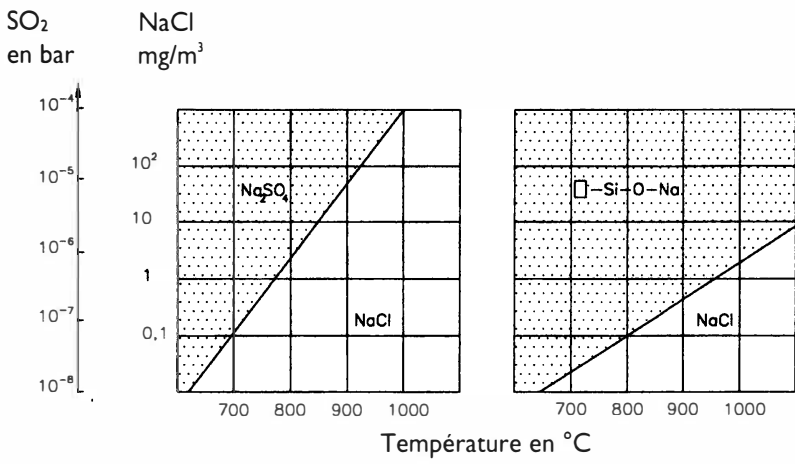

Figure 2 : Diagramme d'équilibre thermodynamique
Une fois formé, le chlore diffuse à travers les couches de sulfate et d'oxyde de fer respectivement pour atteindre la surface externe des tubes où va se dérouler la réaction 2 suivante :

$\mathrm{Fe}+\mathrm{Cl}_{2}(\mathrm{~g}) \longrightarrow \mathrm{FeCl}_{2}$

II se développe ainsi un micro climat corrosif où le chlorure de fer se transforme en oxyde de fer avec régénération de chlore selon la réaction 3 :

$2 \mathrm{FeCl}_{2}+\mathrm{I}, 5 \mathrm{O}_{2}(\mathrm{~g}) \longrightarrow \mathrm{Fe}_{2} \mathrm{O}_{3}+2 \mathrm{Cl}_{2}(\mathrm{~g})$

C'est ce phénomène répétitif de formation-consommation de chlore qui est à l'origine de l'attaque du métal des faisceaux tubulaires comme l'illustre la figure 3 .

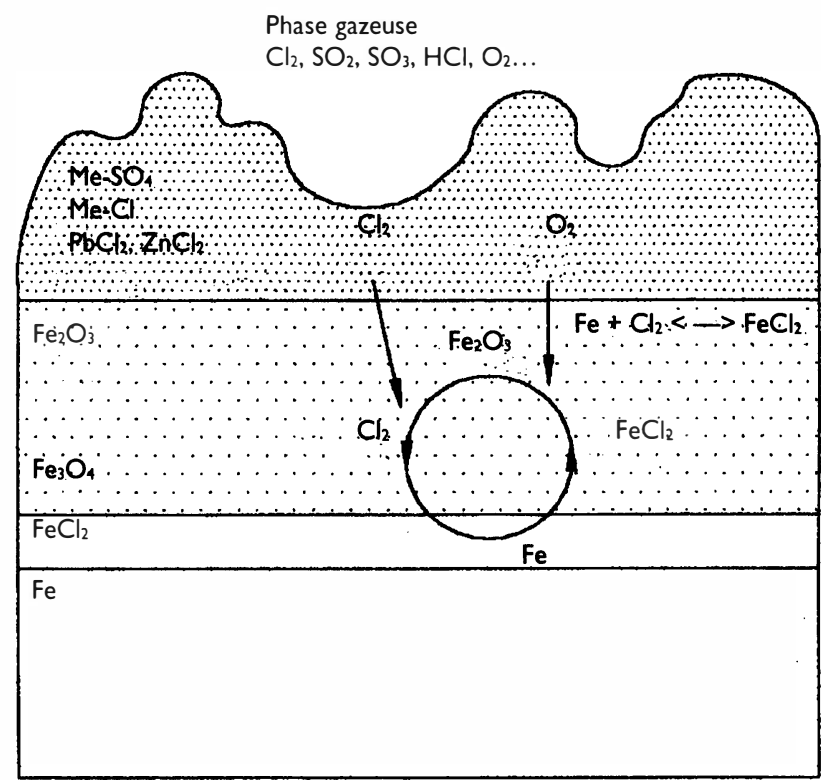

Figure 3 : Schéma de principe du processus de corrosion par le chlore

Un exemple de mécanisme réactionnel décrivant le processus de corrosion et de formation de dépôt dur (dans le cas du chlorure de sodium) est représenté par la figure 4 .

\section{Corrosion du surchauffeur et phénomène d'agglomération des cendres}

Les chlorures métalliques sous forme de particules solides et de vésicules liquides transportées par les fumées ne sont pas sulfatés en phase gazeuse du fait de leurs faibles surfaces spécifiques. On les retrouve sur les surfaces des tubes du surchauffeur où ils viennent adhérer pour réagir avec les dioxydes de soufre pour former des sulfates (le $\mathrm{CaSO}_{4}$ étant prépondérant) de structure cristalline dure et compacte comme le montre la figure 5 .

La conséquence d'un tel phénomène est la formation d'une croûte dure sur la surface des tubes suivi d'un processus de corrosion. On pourra citer à titre d'illustration les mesures d'épaisseur de tubes effectuées à l'usine d'incinération d'or- 


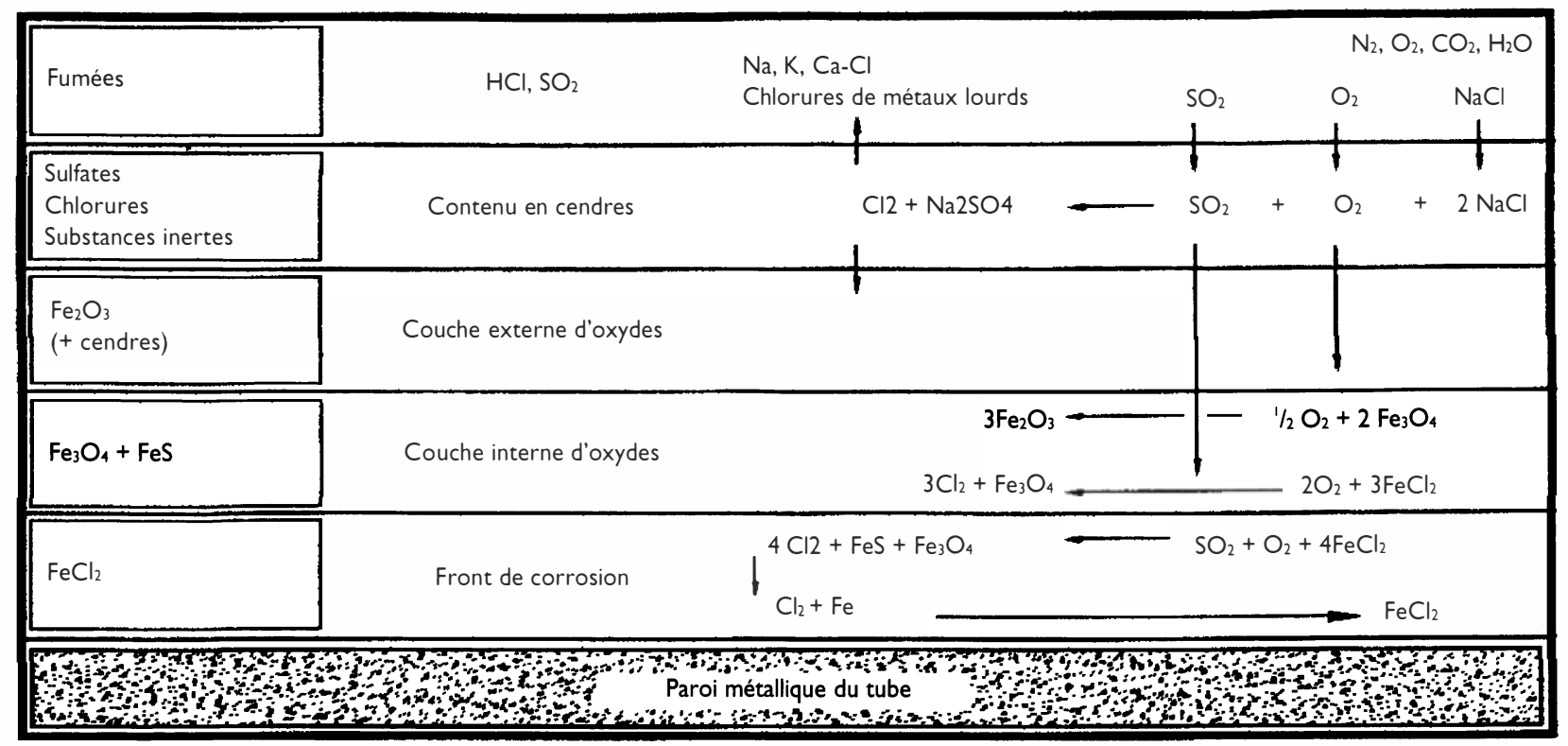

Figure 4 : Exemple de mécanisme réactionnel de corrosion
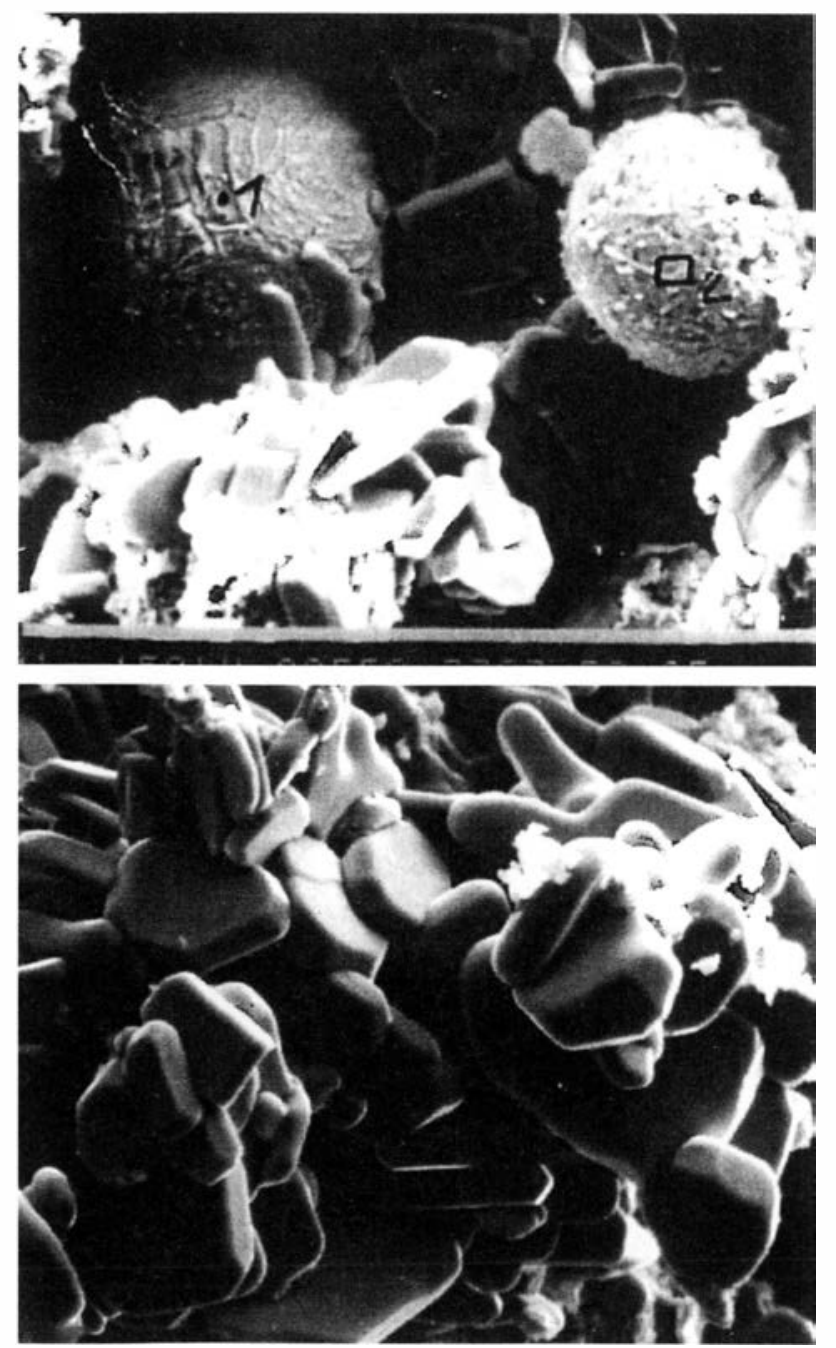

Figure 5 : Transformation de gouttes de chlorure métallique en sulfate dures ménagères de Bremerhaven en Allemagne ${ }^{[2]}$ où des taux de corrosion de plus de $0,5 \mathrm{~mm}$ sur $1000 \mathrm{~h}$ de fonctionnement ont été observés.

\section{LE PROCÉDÉ ICA}

Conscients des problèmes de corrosion dans les chaudières et autres échangeurs de chaleurs équipant les unités d'incinération de déchets, la société Servithen et son commettant la société ICA Chemie proposent un procédé qui permet de réduire considérablement les effets de la corrosion et de diminuer grâce à la formation d'un dépôt meuble de cendre le temps nécessaire au nettoyage des tubes.

Ce procédé agit principalement sur la chimie des gaz, empêchant les réactions néfastes génératrices de chlore gazeux de se dérouler.

\section{Description du procédé}

Le procédé ICA est une technique efficace, présentant nombre d'avantages parmi lesquels on citera l'inhibition des effets néfastes des chlorures métalliques au moment où ils se déplacent dans les gaz de combustion, par injection d'un produit pulvérulent au niveau de l'air secondaire.

Cet agent réactif, appelé ICA 5000 est formé lors de la cuisson à hautes températures d'un minéral (que l'on trouve dans les mines de magnésie) en présence d'acide chlorhydrique. Cette substance est constituée de feuilles de silicate d'aluminium ayant en surface des groupements silanols $(\mathrm{Si}-\mathrm{OH})$ hautement actifs, la dotant ainsi d'une surface spécifique et d'un volume poreux importants.

\section{Caractéristiques physico-chimiques}

Substance pulvérulente de couleur blanche possédant les propriétés suivantes : 
Humidité :

5 à $7 \%$

pH :

Densité apparente :

Granulométrie :

0,4 à $0,5 \mathrm{~g} / \mathrm{cm}^{3}$

$\mathrm{d}_{50}=40 \mu \mathrm{m}$

avec 10 à $20 \%$

de particules

de $\varnothing>63 \mu \mathrm{m}$

Surface spécifique :

$$
400 \mathrm{~m}^{2} / \mathrm{g}
$$

\section{Tableau I : Composition chimique du ICA 5000}

\begin{tabular}{lr}
\hline Composé & \% massique \\
\hline $\mathrm{SiO}_{2}$ & 72,3 \\
\hline $\mathrm{Fe}_{2} \mathrm{O}_{3}$ & 2,8 \\
$\mathrm{Al}_{2} \mathrm{O}_{3}$ & 14,3 \\
\hline $\mathrm{CaO}$ & 0,3 \\
$\mathrm{MgO}$ & 1,1 \\
$\mathrm{Na}$ & 0 \\
$\mathrm{~K}_{2} \mathrm{O}$ & 0,4 \\
\hline Perte au feu à $1000{ }^{\circ} \mathrm{C}$ & 1,8 \\
\hline
\end{tabular}

Il est important de mentionner qu'aucun effet toxique n'a été observé après une série de tests effectués dans des laboratoires de toxicologie.

\section{Mécanisme réactionnel}

Du fait de la présence à la surface de ses particules de groupements silanols hautement actifs, le ICA 5000 réagit avec les chlorures métalliques selon la réaction :

$$
\mathrm{R}-\mathrm{Si}-\mathrm{OH}+\mathrm{MeCl} \rightarrow \mathrm{R}-\mathrm{Si}-\mathrm{O}-\mathrm{Me}+\mathrm{HCl}(\mathrm{g})
$$

Cette réaction présente de nombreux avantages parmi lesquels on citera :

- la formation d'un silicate métallique qui se vitrifie dans la chambre de combustion et vient recouvrir le réfractaire d'une couche protectrice ;

- la formation de $\mathrm{HCl}$, qui à l'opposé du chlore gazeux, ne provoque pas de corrosion dans les conditions opératoires de l'incinération ;

- une partie du ICA 5000 qui ne réagit pas avec les chlorures métalliques se débarrasse de son eau selon la réaction 5 , et se transforme en particules fines de mullite, au point de fusion élevé $\left(1700^{\circ} \mathrm{C}\right)$ qui fixent les particules de cendres pour former des dépôts meubles sur la surface des tubes.

$$
\mathrm{R}-\mathrm{Si}-\mathrm{OH}+\mathrm{R}-\mathrm{Si}-\mathrm{OH} \longrightarrow \underset{\text { (mullite) }}{\mathrm{R}-\mathrm{Si}-\mathrm{O}-\mathrm{Si}-\mathrm{R}+\mathrm{H}_{2} \mathrm{O}}
$$

Ces dépôts sont facilement éliminés par un courant d'air sous pression.

D'un point de vue thermodynamique, la figure 2 montre clairement qu'à équilibre égal de $\mathrm{NaCl}$ (température de $900{ }^{\circ} \mathrm{C}$ et concentration de $\mathrm{NaCl}$ de $10 \mathrm{mg} / \mathrm{m}^{3}$ ), la formation de silicate de sodium est évidente en présence de ICA 5000.

Des analyses chimiques tant sur la phase gazeuse que sur la phase solide ont été réalisées afin de vérifier le mécanisme réactionnel donné par la réaction 4 . La figure 6 montre la présence de sodium dans les fumées de l'usine d'incinération d'Augsburg (Allemagne) détectée par photospectroscopie ${ }^{[3]}$. On peut constater que l'addition de ICA 5000 entraîne une diminution significative de la concentration de sodium.

Des analyses faites sur le dépôt solide après addition de

\begin{tabular}{|c|c|c|c|}
\hline \multicolumn{4}{|c|}{$\begin{array}{c}\text { Tableau } 2 \text { : Analyse quantitative du dépôt } \\
\text { solide prélevé à } 18,5 \text { m de hauteur au niveau } \\
\text { du bloc surchauffeur }\end{array}$} \\
\hline Composés & $\begin{array}{l}\text { ICA } \\
5000\end{array}$ & $\begin{array}{l}\text { Dépôt solide } \\
\text { (en un point) }\end{array}$ & $\begin{array}{l}\text { Dépôt solide } \\
\text { (moyenne) }\end{array}$ \\
\hline $\mathrm{Na}_{2} \mathrm{O}$ & 0,4 & 17,7 & 8,7 \\
\hline $\mathrm{SiO}_{2}$ & 72,3 & 39,8 & 38,1 \\
\hline $\mathrm{Al}_{2} \mathrm{O}_{3}$ & 14,3 & - & 1,8 \\
\hline $\mathrm{CaO}$ & 0,3 & 41,4 & 34,7 \\
\hline $\mathrm{MgO}$ & 1,1 & - & 4,5 \\
\hline $\mathrm{K}_{2} \mathrm{O}$ & 1,8 & - & - \\
\hline $\mathrm{Fe}_{2} \mathrm{O}_{3}$ & 2,8 & 1.1 & 1.5 \\
\hline
\end{tabular}
ICA 5000 (voir tableau 2) montrent d'une part une augmentation de la composition d'oxydes de sodium, calcium et magnésium et d'autre part une diminution de la compo-

Analyse photo-spectrométrique du $\mathrm{Na}$ effectuée dans la chambre de combustion à une hauteur de $13,4 \mathrm{~m}$

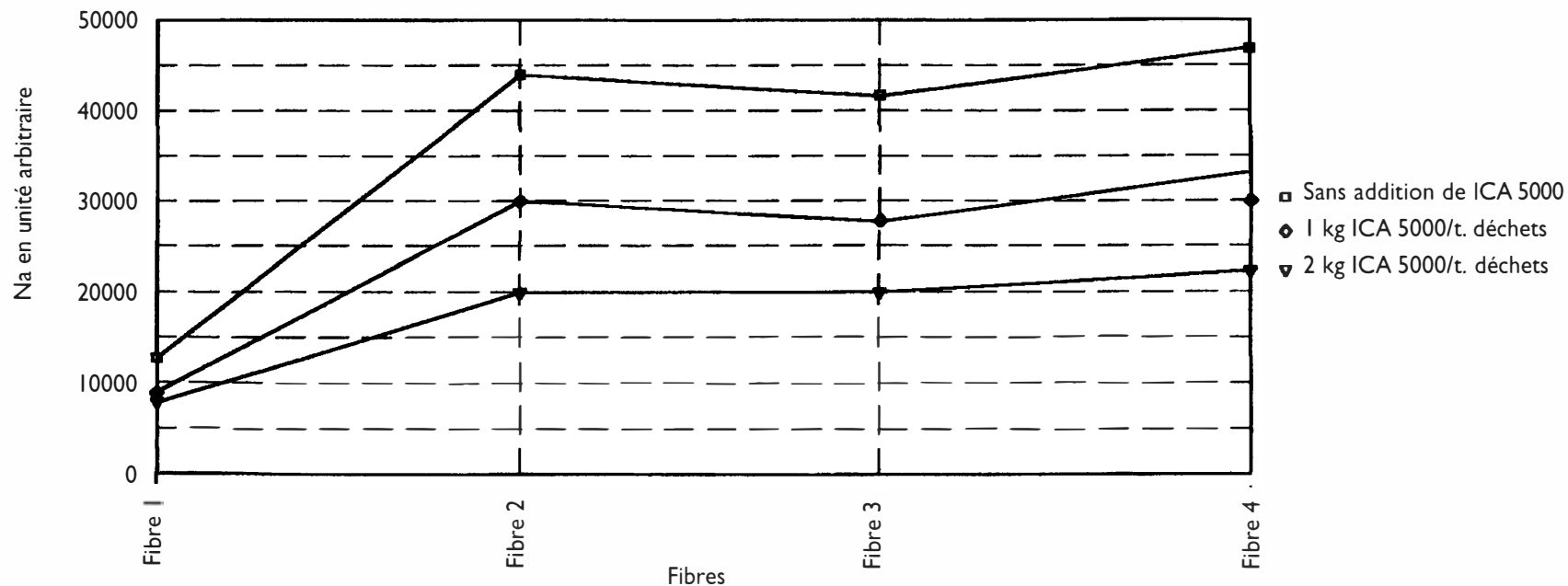

Figure 6 : Analyse spectrophotométrique du sodium dans les fumées 
sition en silice et alumine. Cette augmentation de la teneur en sodium en phase solide explique sa diminution en phase gazeuse.

Il a été constaté par ailleurs, une augmentation du débit de $\mathrm{NaOH}$ nécessaire à la neutralisation des gaz acides. Dans le cas de l'usine de Bremerhaven, on passe de 2,6 litres (en absence de ICA 5000) à 3,2 litres de $\mathrm{NaOH}$ par tonne de déchets traités. Cette augmentation est attribuée à la présence dans les effluents gazeux à traiter du $\mathrm{SO}_{2}$ qui devait réagir avec les chlorures métalliques en absence de ICA 5000 .

Ces différents résultats d'analyse sont la preuve de la grande affinité que possède ICA 5000 vis-à-vis des chlorures métalliques.

\section{Alimentation}

Le ICA 5000 est injecté au moyen d'air comprimé dans le collecteur d'air secondaire du four et est distribué ainsi de manière régulière dans la chambre de combustion comme le montre la figure 7. L'installation d'un équipement approprié d'injection est nécessaire (par exemple : big-bag, vis sans fin, venturi, et accessoires).

La consommation en ICA 5000 est de l'ordre de I kg/tonne de déchets. Le dosage peut se faire en intervenant sur la vis d'alimentation soit manuellement, soit automatiquement par mesure spectroscopique de la concentration en alcali dans les gaz au niveau de la chambre de combustion.

II est intéressant de signaler par ailleurs, que ce produit peut également être injecté en émulsion, mélangé par exemple aux eaux usées récupérées au niveau des décharges d'ordures.

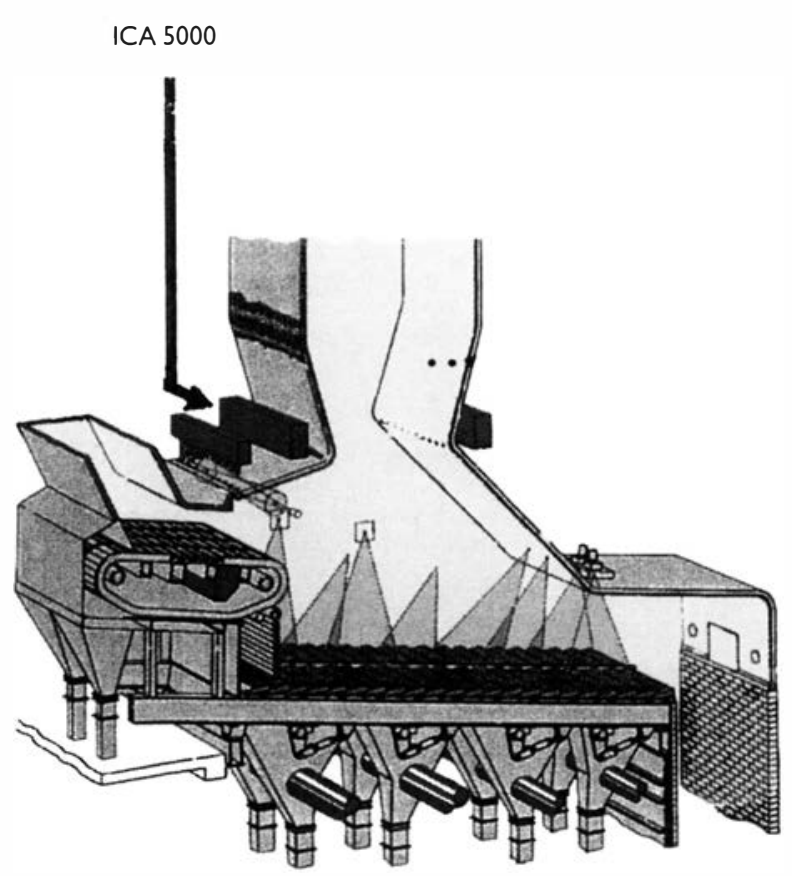

Figure 7 : Injection de ICA 5000 au niveau de l'air secondaire

\section{Résultats obtenus}

Le ICA 5000 est utilisé industriellement depuis mars 1996 dans différentes usines d'incinération d'ordures ménagères en Europe. Des résultats extrêmement positifs ont été obtenus depuis le début. A titre d'exemple, on citera ceux de l'usine de Bremerhaven en Allemagne:

- Taux de corrosion pratiquement nul au terme de $22000 \mathrm{~h}$ de fonctionnement alors que des taux de $0,5 \mathrm{~mm} / 1000 \mathrm{~h}$ de fonctionnement avaient été observés avant l'utilisation de cet additif, entraînant des dépenses régulières importantes pour le remplacement des faisceaux de tubes.

- Diminution du temps nécessaire au nettoyage des surfaces de tubes, entraînant un accroissement du temps de fonctionnement et par là même de la quantité de déchets traités. En effet, on passe de 300000 tonnes à 317000 tonnes/an, soit une augmentation de $5,7 \%$.

- Diminution des frais d'entretien, puisqu'on passe de 70 DM à $60 \mathrm{DM} /$ tonne, soit une réduction de $14,3 \%$.

\section{Perspectives}

Une version plus développée (ICA 6000) et possédant les mêmes propriétés que l'ICA 5000, est à l'heure actuelle au stade d'expérimentation. Cette substance a la particularité d'avoir une structure des pores et une distribution du diamètre de pores (voir figure 8 ) qui lui confère, en plus de l'effet inhibiteur de corrosion, un pouvoir d'adsorption vis-àvis des dioxines, furanes et métaux lourds. Une telle substance pourrait se substituer au charbon actif.

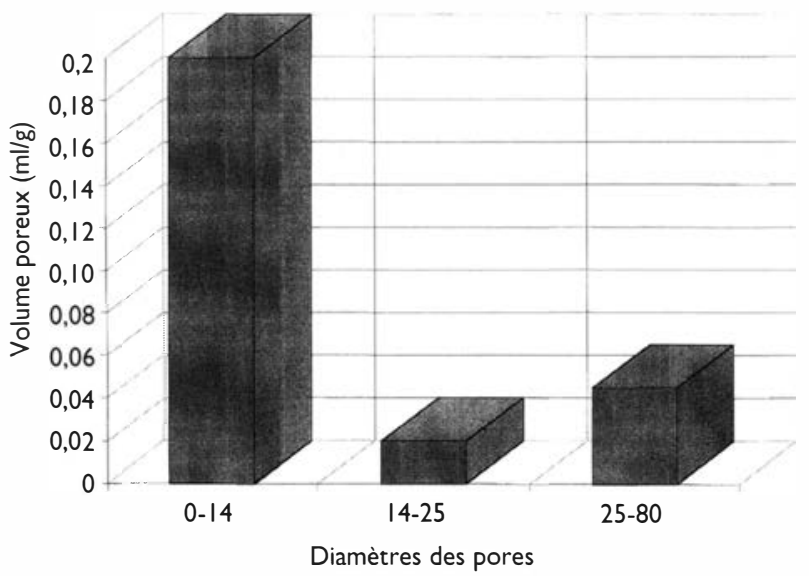

Figure 8 : Courbe de distribution de diamètre des pores pour I'ICA 6000

\section{Données économiques}

Une estimation de l'investissement nécessaire et du profit réalisé suite à l'utilisation du procédé ICA a été faite sur la base des données de l'usine de Bremerhaven. Cette estimation représentée par la figure 9 prend en considération le coût d'achat du produit ICA 5000, l'investissement initial de l'installation d'injection, le coût correspondant à l'aug- 


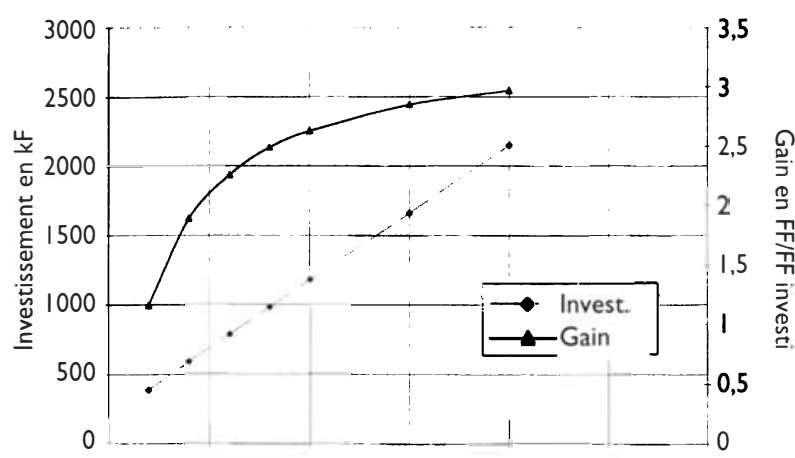

Figure 9 : Investissement et gain réalisés en fonction de la capacité de l'usine

mentation du débit de soude pour le lavage des gaz et le coût relatif à la prise en charge de l'augmentation de la masse des sous produit (Refiom) après traitement des gaz. On peut

constater que le gain réalisé augmente avec la capacité de l'usine.

\section{* K.-M. Allal,}

Servithen - Les Hauts de Villancy - 8, rue Guillaume de Bois Nivard - 78640 Neauphle-le-Château

\section{** F. Birkner}

ICA Chemie GesmbH \& Co KG - A-3384 Groß - Sierming 33 - Autriche

\section{Bibliographie}

[I] W.Spiegel, E.Barth, H.Wagner, Damaging process at refractory linings in waste incineration plants, VGB Power Technologie, Oct. 1998.
[2] F.Birkner, Rapport interne sur ICA 5000,1998

[3] E. Barth, H. Heinz, Primärmaßnamen zur Reduzierung von Korrosionnen und..., VGB KraftwerksTechnik, Nov. 1998.

\section{Stabilisation des Déchets et Environnement 99}

\section{Waste Stabilisation and Environment 99}

Actes du congrès des 13-16 avril 1999 - Lyon-Villeurbanne, France

Thème I

Déchets stabilisés : rétention et émission des polluants

Thème II Transfert et évolution dans l'environnement des polluants issus des déchets stabilisés

Thème III Impact potentiel des déchets stabilisés sur la santé et l'environnement

Thème IV Approches intégrées - Application à l'alternative entre stockage et utilisation

Conférences invitées et sessions parallèles

Atelier I Gestion des résidus d'incinération des ordures ménagères

Atelier II Valorisation des résidus de process thermiques (cendres volantes de charbon...)

Atelier III Étude des matrice cimentières

Atelier IV Réglementation, normalisation,

outils d'évaluation

Format $14,5 * 21$ - 1999 - 504 pages : 580 F TTC franco

\section{Posters du congrès}

Format $14,5 * 21$ - 1999 - 264 pages : 270 F TTC franco

\section{Actes + posters : 800 F TTC franco}

Règlement à la commande

Société Alpine de Publications (SAP)

7, chemin de Gordes - 38100 Grenoble

Tél. : 0476432864 - Fax : 0476569409 - E.et.T@wanadoo.fr 